\title{
Fatal viral infection-associated encephalopathy in two Chinese boys: a genetically determined risk factor of thermolabile carnitine palmitoyltransferase II variants
}

\author{
Chloe Miu Mak ${ }^{1}$, Ching-wan Lam², Nai-chung Fong ${ }^{3}$, Wai-kwan Siu ${ }^{1,4}$, Han-chih Hencher Lee ${ }^{1}$, \\ Tak-shing Siu ${ }^{5}$, Chi-kong Lai ${ }^{1}$, Chun-yiu Law ${ }^{1}$, Sui-fun Tong ${ }^{2}$, Wing-tat Poon ${ }^{1}$, David Shu-yan Lam ${ }^{6}$, \\ Ho-leung $\mathrm{Ng}^{4}$, Yuet-ping Yuen ${ }^{1,7}$, Sidney Tam ${ }^{5}$, Tak-lun Que ${ }^{4}$, Ngai-shan Kwong ${ }^{6}$ and Albert Yan-wo Chan ${ }^{1}$
}

Influenza-associated encephalopathy (IAE) is a potentially fatal neurological complication of influenza infection usually in the presence of high and persistent fever. Thermolabile carnitine palmitoyltransferase II enzyme (CPT-II) predisposes IAE, so far only described in Japanese. As the genetic origins of Japanese and Chinese are alike, similar genetic risk factors in CPT-II are expected. We report the first two unrelated Chinese patients of thermolabile CPT-II variants that underlain the persistent high fever-triggered viral infection-associated encephalopathy, multi-organ failure and death. Elevated (C16:0+C18:1)/C2 acylcarnitines ratio and the CPT2 susceptibility variant allele [p.Phe352Cys; p.Val368lle] were detected. The asymptomatic family members of one patient also had abnormal long-chain acylcarnitines. In our experience of biochemical genetics, the elevated (C16:0+C18:1)/C2 acylcarnitines ratio is unusual and specific for thermolabile CPT-II variants. Allele frequency of [p.Phe352Cys; p.Val368Ile] among Hong Kong Chinese was 0.104, similar to Japanese data, and [p.Phe352Cys] has not been reported in Caucasians. This may explain the Asian-specific phenomenon of thermolabile CPT-II-associated IAE. We successfully demonstrated the thermolabile CPT-II variants in patients with viral infection-associated encephalopathy in another Asian population outside Japanese. The condition is likely under-recognized. With our first cases, it is envisaged that more cases will be diagnosed in subsequent years. The exact pathogenic mechanism of how other factors interplay with thermolabile CPT-II variants and high fever leading to IAE, is yet to be elucidated. Fasting and decreased intake during illness may aggravate the disease. Further studies including high risk and neonatal screening are warranted to investigate its expressivity, penetrance and temperature-dependent behaviors in thermolabile CPT-II carriers. This may lead to discovery of the therapeutic golden window by aggressive antipyretics and L-carnitine administration in avoiding the high mortality and morbidity of IAE. Journal of Human Genetics (2011) 56, 617-621; doi:10.1038/jhg.2011.63; published online 23 June 2011

Keywords: Chinese; Coxsackie virus; H1N1 human swine influenza; influenza-associated encephalopathy; thermolabile carnitine palmitoyltransferase II; viral infection-associated encephalopathy

\section{INTRODUCTION}

Influenza-associated encephalopathy (IAE) is a highly fatal neurological complication of influenza infection. It is characterized by sudden onset of high fever, recurrent convulsions, early development of coma, multiple-organ failure and death usually within $2-3$ days. IAE is much more common in Japanese than in Caucasians, implying possibly a genetic difference. Chen et al. ${ }^{1}$ first conducted a 3 -year investigation on 34 previously healthy Japanese patients diagnosed with IAE. In their study, a significant portion of patients suffering from severe IAE showed abnormal acylcarnitine pattern indicating a failing mitochondrial fatty acid oxidation. The long-chain acylcarnitines were elevated with the characteristic ratio of $(\mathrm{C} 16: 0+\mathrm{C} 18: 1) / \mathrm{C} 2$ ratio greater than 0.09 during febrile episode (normal $<0.048$ ). The authors further demonstrated a reduced enzyme activity of carnitine palmitoyltransferase II (CPT-II, MIM $\left.{ }^{\star} 600650\right)$ at high temperature in patients with genotype of [p.Phe352Cys; p.Val368Ile]. Shinohara et al. ${ }^{2}$ confirmed the similar findings in 29 Japanese patients with acute encephalopathy irrespective of the types of preceding infections.

A comprehensive functional study documented the defective enzymatic kinetics and the associated dominant negative effect on the homotetrameric CPT-II enzyme in three thermolabile CPT-II variants. ${ }^{3}$ The three variants are [p.Phe352Cys; p.Val368Ile], [p.Pro504Leu; p.Val605Leu] and

\footnotetext{
${ }^{1}$ Chemical Pathology Laboratory, Department of Pathology, Princess Margaret Hospital, Hong Kong SAR, China; ${ }^{2}$ Department of Pathology, Li Ka Shing Faculty of Medicine, The University of Hong Kong, Hong Kong SAR, China; ${ }^{3}$ Department of Paediatrics and Adolescent Medicine, Princess Margaret Hospital, Hong Kong SAR, China; ${ }^{4}$ Department of Clinical Pathology, Tuen Mun Hospital, Hong Kong SAR, China; ${ }^{5}$ Division of Clinical Biochemistry, Queen Mary Hospital, Hong Kong SAR, China; ${ }^{6}$ Department of Paediatrics and Adolescent Medicine, Tuen Mun Hospital, Hong Kong SAR, China and ${ }^{7}$ Department of Chemical Pathology, Prince of Wales Hospital, Hong Kong SAR, China Correspondence: Dr C-W Lam, Department of Pathology, Li Ka Shing Faculty of Medicine, The University of Hong Kong, Hong Kong SAR, China. E-mail: ching-wanlam@pathology.hku.hk
}

Received 3 February 2011; revised 9 March 2011; accepted 11 May 2011; published online 23 June 2011 
[p.Phe352Cys; p.Val368Ile; p.Val605Leu]. Recently, Kubota et al. ${ }^{4}$ reported reduced blood ATP levels and systemic mitochondrial dysfunction in thermolabile CPT-II patients with acute encephalopathy and febrile seizures. They postulated that thermolabile CPT-II is causally related to the severity of encephalopathy in Japanese population. As the genetic backgrounds between Japanese and Chinese are alike, ${ }^{5}$ the phenomenon of thermolabile CPT-II-associated IAE in Chinese is expected. Recently, two Hong Kong Chinese boys unfortunately died of viral infectionassociated encephalopathy and we genetically confirmed thermolabile CPT-II variants together with abnormal acylcarnitine profiles.

\section{CASE HISTORY}

\section{Patient A}

Patient A was a 4-year-old Chinese boy who was admitted for high fever of $42{ }^{\circ} \mathrm{C}$ with productive cough and decrease in playfulness. He suffered from abrupt onset of generalized clonic-tonic convulsion for $25 \mathrm{~min}$ soon after the admission. He also had metabolic acidosis ( $\mathrm{pH} 7.26$, base excess $-19.5 \mathrm{mmoll}^{-1}$ ) and hypoglycemia (glucose $\left.1.3 \mathrm{mmoll}^{-1}\right)$. Creatine kinase was $8884 \mathrm{Ul}^{-1}$ and troponin I was $3.11 \mu \mathrm{g} \mathrm{l}^{-1}$. Viral culture of his nasopharyngeal aspirate subsequently showed Coxsackie virus group A. Computed tomography of brain revealed cerebral edema (Figure 1). Because of his rapid deterioration, metabolic work-up was done. The condition worsened with multiorgan failure and the patient unfortunately succumbed on the 25th day after admission despite intensive management. Patient A had a past medical history of dyskinetic cerebral palsy, global developmental delay and bilateral sensorineural hearing loss. Initial metabolic workup at 1 year of age including plasma ammnonia $\left(48 \mu \mathrm{moll}^{-1}\right)$, plasma lactate $\left(1.4 \mathrm{mmoll}^{-1}\right)$, plasma amino acids, urinary broad spectrum metabolic screening for disorders of organic acids, mono- and di-saccharides as well as purines and pyrimidines were negative. There was no plasma acylcarnitines performed during that time.

\section{Patient B}

Patient B was a 3-year-old boy with good past health and normal development. He was admitted on the third day of fever with

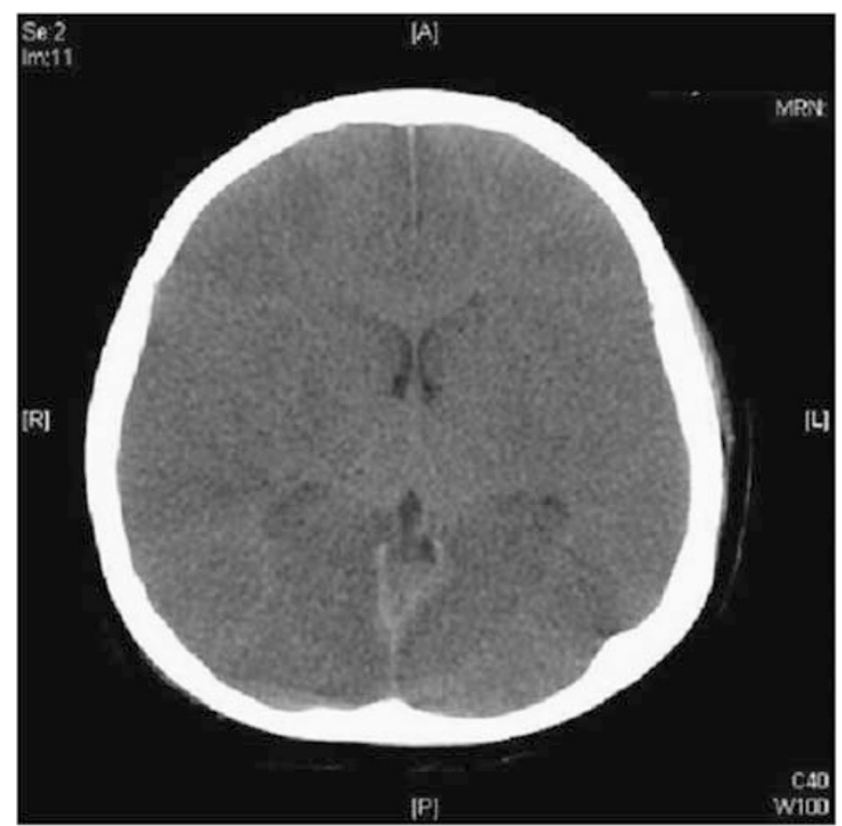

Figure 1 The image of computed tomography of brain of the patient $A$ showed cerebral edema. maximum temperature at $41^{\circ} \mathrm{C}$. The nasopharyngeal aspirate was positive for influenza A virus subtype $\mathrm{H} 1$ (human swine influenza) by reverse transcriptase polymerase chain reaction. There were two episodes of abrupt onset convulsion after admission. He underwent a rapid deterioration with shock and disseminated intravascular coagulation. He was on critical condition requiring ventilator and multiple inotropic support. Computed tomography of brain showed bilateral cerebral edema and hypodense brainstem with coning. The patient suffered from multi-organ failure and unfortunately died on the fourth day of admission. Chemical Pathology consultation was received and investigation for influenza-associated encephalopathy caused by thermolabile carnitine palmitoyltransferase II (CPT-II) variant was initiated immediately. DNA sequencing of the viral specimen performed by another laboratory also confirmed no mutant strain of human swine influenza.

\section{Genetic and biochemical analysis of thermolabile CPT-II variant} subjects

Informed consent was obtained with genetic counseling provided for all tested individuals. Local ethic approval was documented. Both patients were heterozygous for c.1055T $>\mathrm{G}$ [p.Phe352Cys] and homozygous for c.1102G $>$ A [p.Val368Ile] by PCR and direct sequencing with genomic DNA. Primer sequences and protocol are available upon request. In other words, they were compound heterozygous for [p.Phe352Cys; p.Val368Ile]+[p.Val368Ile]. The allele [p.Phe352Cys; p.Val368Ile] is CPT-II susceptible. No CPT2 mutation that leads to classical CPT-II deficiency was identified. The past medical problem of patient A cannot be explained by the inheritance of thermolabile CPT-II variant.

Patient A showed elevations of plasma long-chain acylcarnitines, mainly as C18:1-acylcarnitine by qualitative assay. The blood sample was taken at body temperature of $37^{\circ} \mathrm{C}$ while the patient was on multiple inotropes. Unfortunately, there was no residual sample left for quantitative acylcarnitine measurement. In patient B, quantitative plasma acylcarnitines were performed on the retrospective sample collected on the second day for other tests. $(\mathrm{C} 16: 0+\mathrm{C} 18: 1) / \mathrm{C} 2$ ratio was transiently elevated to 0.057 (normal $<0.048$ ).

Family screening was conducted. The father and 10-year-old elder sister of patient $\mathrm{A}$ and the mother of patient $\mathrm{B}$ shared the same genotype as the probands, viz. [p.Phe352Cys; p.Val368Ile]+ [p.Val368Ile]. The mother of patient A and the father of patient B were homozygous for p.Val368Ile only. Figure 2 shows the family pedigree with their genotypes and biochemical results. Table 1 shows the plasma acylcarnitine profiles of the subjects. The (C16:0+C18:1)/ $\mathrm{C} 2$ ratios of the father and the elder sister of patient $\mathrm{A}$ and the mother of patient B were all elevated: 0.224, 0.111 and 0.048, respectively, (normal < 0.048). Ratio of the mother of patient A and the father of patient $\mathrm{B}$ was 0.183 and 0.037 , respectively. They were all along healthy. The parents were non-consanguineous. Parents of patient A shared the same homeland in Jiangmen Guangdong, China.

\section{DISCUSSIONS}

IAE is a fatal neurological complication of high-fever influenza infection. Thermolabile CPT-II enzyme predisposes IAE, so far only reported in Japanese. We report the first two Chinese cases of thermolabile CPT-II variants which underlain the persistent high fever-triggered viral infection-associated encephalopathy, multi-organ failure and death in two Hong Kong Chinese boys. Elevated (C16:0+C18:1)/C2 ratio and the CPT2 susceptibility variants [p.Phe352Cys; p.Val368Ile] were detected. In our patients, one had Coxsackie infection and the other had H1N1 swine influenza. The crucial risk is high fever, which paralyzes the thermolabile CPT-II enzyme leading to a deadly mitochondrial failure. 
a
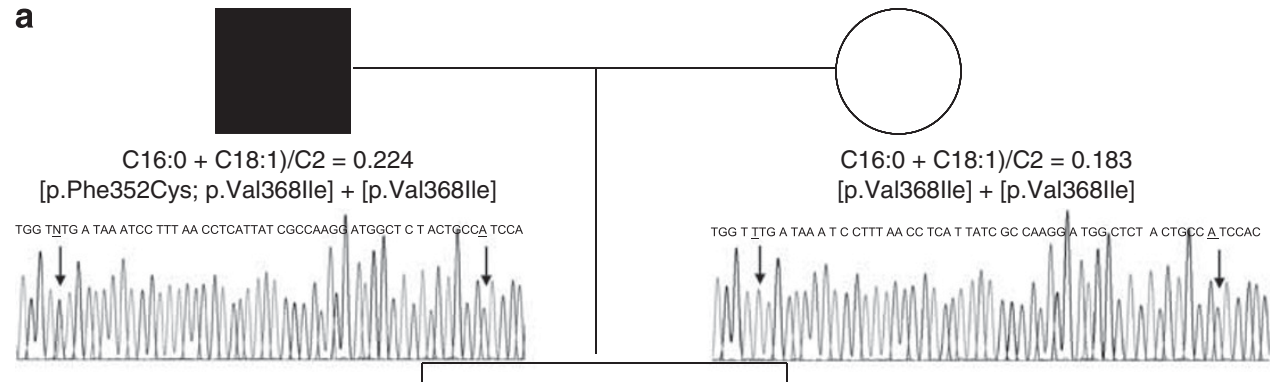
[p.Phe352Cys; p.Val368lle] + [p.Val368lle]
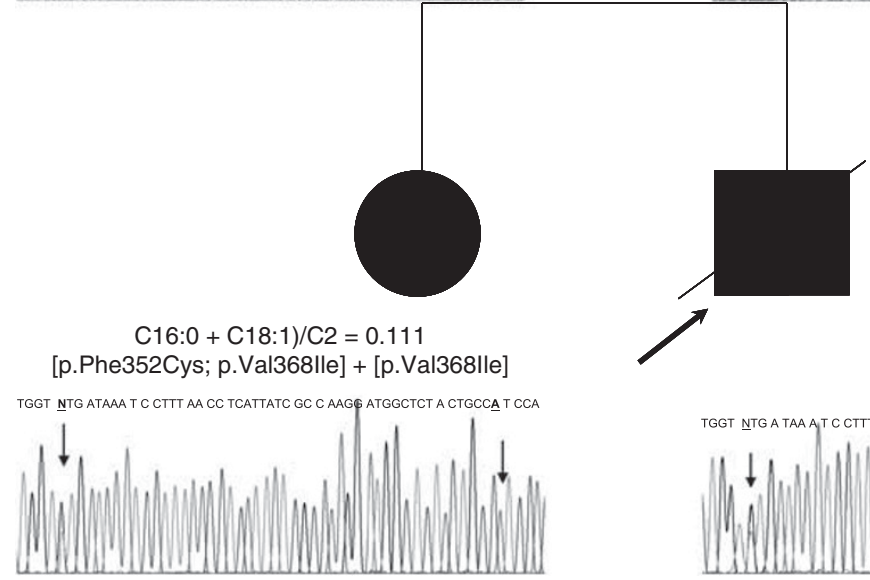

Elevated $\mathrm{C} 18: 1$ by qualitative assay only [p.Phe352Cys; p.Val368lle]+ [p.Val368lle]

b

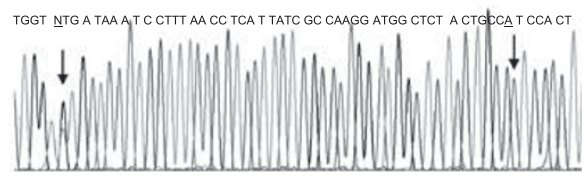

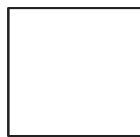

$\mathrm{C} 16: 0+\mathrm{C} 18: 1) / \mathrm{C} 2=0.037$ $[p . V a l 368 \| l e]+[p . V a l 368 \| l e]$

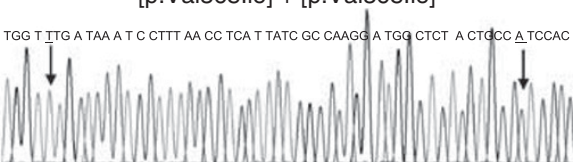

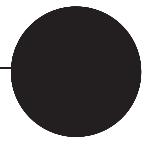

$\mathrm{C} 16: 0+\mathrm{C} 18: 1) / \mathrm{C} 2=0.048$ [p.Phe352Cys; p.Val368lle] + [p.Val368lle]

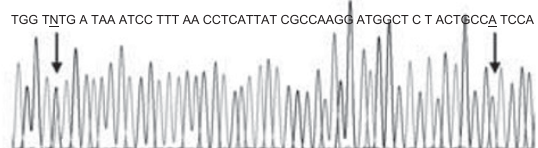

$\mathrm{C} 16: 0+\mathrm{C} 18: 1) / \mathrm{C} 2=0.057$

[p.Ph352Cys; p.Val368II ] + [p.Val368II]

Figure 2 The family pedigrees of (a) patient A and (b) patient B with their genotypes (in sense direction) and biochemical results are shown.

Interestingly, the asymptomatic family members carrying the CPT2 susceptibility variants [p.Phe352Cys; p.Val368Ile] also showed abnormally elevated acylcarnitine ratio at normal body temperature. They were all along healthy and recalled no previous episodes of persistent high fever. This echoes the same finding described by Chen et al. ${ }^{1}$

Moreover, the enzymatic activity of heterozygous p.Val368Ile CPTII variant was reduced to $91 \%$ at $41^{\circ} \mathrm{C} .{ }^{1}$ The variant p.Val368Ile alone is not classified as thermolabile CPT-II variants by Yao et al..$^{3}$ Intriguingly, the mother of patient A homozygous for p.Val368Ile also had a high ratio of 0.183 when compared with the mother reported in Chen's paper who was only heterozygous for p.Val368Ile and had a normal ratio (0.016). This probably suggests a more deleterious effect of homozygous p.Val368Ile variant.

In our local experience of analyzing acylcarnitine profiles in more than 100 high-risk patients suspected of inborn errors of metabolism, none of them showed abnormal $(\mathrm{C} 16: 0+\mathrm{C} 18: 1) / \mathrm{C} 2$ ratio. The presence of abnormal ratio is very unusual and is a characteristic of mitochondrial fatty acid oxidation dysfunction due to thermolabile CPT-II variants. In addition, we observed that the (C16:0+C18:1)/C2 
Table 1 Plasma acylcarnitine results of the patients and their family members

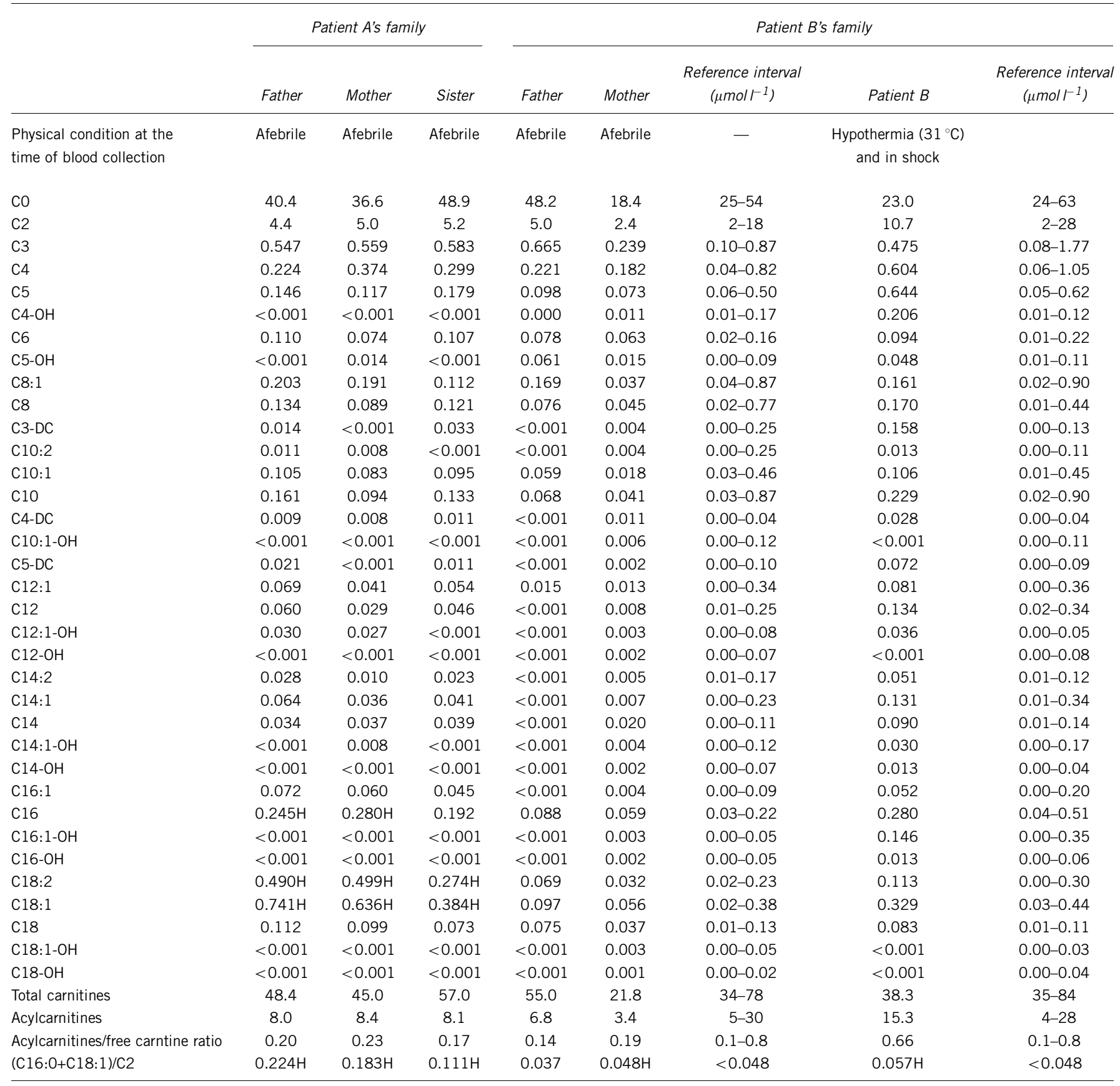

Abbreviation: $\mathrm{H}$, high.

ratio is more sensitive than the absolute concentrations of the individual long-chain acylcarnitines. CSF acylcarnitines may be more helpful than plasma acylcarnitines. However, the genotype did not appear to correlate exactly with the biochemical abnormalities or the clinical phenotypes in all subjects. Patient B had high fever but only elevated $(\mathrm{C} 16: 0+\mathrm{C} 18: 1) / \mathrm{C} 2$ ratio, whereas the asymptomatic family members of patient A showed remarkably abnormal longchain acylcarnitines. There may be other factors interplaying with the pathogenic mechanism of IAE. Fasting and decrease in intake during illness likely further aggravate the disease. The time length of persistent high fever may also be critical for the disease severity. More families should be examined to demonstrate the association between the thermolabile CPT-II genotypes and the concentrations of the longchain acylcarnitines in Chinese population.

The population frequencies of p.Val368Ile; c.1102G > A (rs1799821) are similar between alleles $\mathrm{G}$ and $\mathrm{A}$ in Caucasians, Japanese and Chinese. This probably signifies a more benign nature of p.Val368Ile in concordance with the functional findings by Chen et al. ${ }^{1}$ and Yao et al. ${ }^{3}$ Intriguingly, from the Ensembl database (http:// www.ensembl.org, accessed on 9 March 2011), all Northern and Western European carry the wild-type allele (p.Phe352; c.1055T) with allele frequency of 1.000 . In contrast, variant allele G (p.Phe352Cys; c.1055G, rs2229291) is much more prevalent among Japanese and Chinese with allele frequencies ranging from 0.151 to 
0.205. Conceivably, the frequent combination of [p.Phe352Cys; p.Val368Ile] dictates the genetic differences and so may explain the higher prevalence and Asian-specific phenomenon of thermolabile CPT-II-associated IAE in Japanese and Chinese. We also screened 178 unrelated Hong Kong Chinese healthy subjects and 37 of them carried the same genotype as our patients (that is [p.Phe352Cys; p.Val368Ile] $+[$ p.Val368Ile]). In other words, the allele frequency of the thermolabile CPT-II variant [p.Phe352Cys; p.Val368Ile] is 0.104 among Hong Kong Chinese. Diagnosis of thermolabile CPT-II IAE is likely largely missed.

We successfully demonstrated the thermolabile CPT-II variants in patients with viral infection-associated encephalopathy in another Asian population outside Japanese. We report the first two Chinese patients of thermolabile CPT-II variants which underlain the fatal viral infection-associated encephalopathy. These variants do not cause classical CPT-II deficiency, and patients harboring any of these variants are asymptomatic most of the time. However, they are prone to viral infection (high fever)-related encephalopathy complicated with multi-organ failure and high risks of morbidity and mortality. A timely and accurate genetic diagnosis of thermolabile CPT-II is important when aggressive antipyretic therapy as well as intravenous administration of L-carnitine might be helpful in these patients. Our cases illustrate that the association of serious encephalopathy and impaired mitochondrial fatty acid oxidation is not only seen in influenza infection, but also in other viral infections with high fever. The prevalence of thermolabile CPT-II variants among Chinese and Japanese is relatively common. Further studies are warranted to investigate its expressivity, penetrance and temperature-dependent behaviors in more thermolabile CPT-II carriers and IAE patients in Asia. This may lead to the discovery of therapeutic golden window by aggressive antipyretics and L-carnitine administration to avoid the high mortality and morbidity. This also poses significant consideration in the management of viral infection associated with high fever among Asian children, and the need of biochemical and genetic screening in at risk children.

1 Chen, Y., Mizuguchi, H., Yao, D., Ide, M., Kuroda, Y., Shigematsu, Y. et al. Thermolabile phenotype of carnitine palmitoyltransferase II variations as a predisposing factor for influenza-associated encephalopathy. FEBS Lett. 579, 2040-2044 (2005).

2 Shinohara, M., Saitoh, M., Takanashi, J. I., Yamanouchi, H., Kubota, M., Goto, T. et al. Carnitine palmitoyl transferase II polymorphism is associated with multiple syndromes of acute encephalopathy with various infectious diseases. Brain Dev. 33, 512-517 (2011)

3 Yao, D., Mizuguchi, H., Yamaguchi, M., Yamada, H., Chida, J., Shikata, K. et al. Thermal instability of compound variants of carnitine palmitoyltransferase II and impaired mitochondrial fuel utilization in influenza-associated encephalopathy. Hum. Mutat. 29, 718-727 (2008).

4 Kubota, M., Chida, J., Hoshino, H., Ozawa, H., Koide, A., Kashii, H. et al. Thermolabile CPT II variants and low blood ATP levels are closely related to severity of acute encephalopathy in Japanese children. Brain Dev. (e-pub ahead of print 27 January 2011)

5 Lam, C. W. Origin of the Japanese population. Science 284, 1125 (1999) 